\title{
Progress of Optical Fiber Sensors and Its Application in Harsh Environment
}

\author{
Min ZHANG, Xiaohong MA, Liwei WANG, Shurong LAI, Hongpu ZHOU, \\ Huafeng ZHAO, and Yanbiao LIAO
}

\author{
Optical Fiber Sensors Lab, EE Dept. Tsinghua University, 100084 Beijing, China \\ *Corresponding author: Min ZHANGＥ-mail: minzhang@mail.tsinghua.edu.cn
}

\begin{abstract}
Fiber sensors have been developed for industry application with significant advantages. In this paper, Fiber sensors for oil field service and harsh environment monitoring which have been investigated in Tsinghua University are demonstrated. By discussing the requirements of practical applications, the key technologies of long-period fiber grating (LPFG) based fiber sensor, optical spectrum analyzer for oil detection, laser induced breakdown spectroscopy (LIBS) system for soil contamination monitoring, and seismic sensor arrays are described.
\end{abstract}

Keywords: Fiber sensor, seismic geophone, gas sensor, spectrum analyzer, LIBS

\section{Introduction}

With the development of opto-electronical technologies and the increasing requirements in industry and national defense, optical fiber sensors have been widely accepted and used in the past 10 years. Especially for those applications in harsh environment, such as oil field service, civil engineering, navigation, and monitoring in ocean, the advantages of fiber sensors are significant: immune to electromagnetic interference(EMI), small size, and easy for network integration. The requirement of fiber sensors for harsh environment increases rapidly, hence the market of fiber sensors grows faster. Among these practical applications, fiber geophones for ocean seismicity measurement, Raman optical time domain reflectometer (ROTDR) for down well distributed temperature sensing and distributed vibration sensing systems for petroleum pipeline protection have occupied most of fiber sensor market. The microstructure fiber sensors which are fabricated in fiber to be used in small and cramped space also have attracted the research groups all over the world. For these reasons, we have focused on laser induced breakdown spectroscopy(LIBS) system, down well spectrum analyzer, fiber acoustic sensing system, and long-period fiber grating(LPFG) based sensors since 2002. In this paper, we will discuss the key technologies of those fiber sensors. Gas sensors and humidity sensors by the use of LPFG will be discussed in Section 2.1, an optical spectrum analyzer for down well oil detection will be discussed in Section 2.2, LIBS for soil contamination monitoring will be discussed in Section 2.3, and optical fiber seismic geophone systems will be shown in Section 3.

\section{Optical fiber sensors for harsh environment}

\subsection{LPFG based sensors}

An LPFG can be fabricated by periodically

Received: 21 September 2010 / Revised version: 18 October 2010

C The Authors(s) 2010.This article is published with open access at Springerlink.com 
modulating the refractive index of the cladding or the core of a single-mode optical fiber with $\mathrm{CO}_{2}$ laser as shown in Fig. 1 which was first used by Rao et al.[1]. The resonant wavelength and amplitude of the attenuation bands of an LPFG are very sensitive to perturbations of external surroundings, and thus LPFGs can be used to measure not only temperature and strain, but also the solution concentration or $\mathrm{pH}$ value by changing the refractive index surrounding LPFG. In recent years a lot of researches have focused on how to enhance sensor's sensitivity to ambient refractive index changes by cascaded LPFGs and high-refractive-index coated LPFGs.

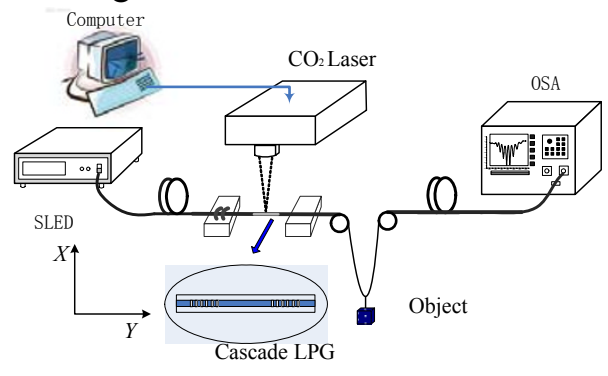

Fig. 1 Setup of LPFG fabrication.

When two LFPGs are connected in series in one optical fiber with a short distance of few centimeters, we can obtain cascaded LFPGs featuring a Mach-Zehnder interferometer as shown in Figs. 2 and 3. The transmission spectrum of cascaded LFPGs shown in Fig. 3 contains several sinusoidal interference fringes exhibiting an envelope corresponding to the attenuation bands normally presented for single LFPG[2-3].

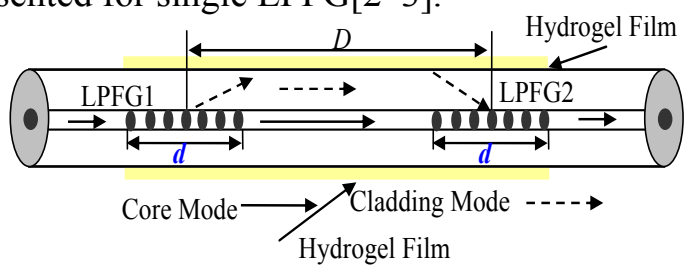

Fig. 2 Principle of cascaded LFPGs.

When being coated with hydrogel or palladium silver (Pd-Ag) film, the cascaded LFPGs can be used as relative humidity $(\mathrm{RH})$ sensors or hydrogen $\left(\mathrm{H}_{2}\right)$ sensors respectively, which is useful for strategic weapon storage where the space is airtight and cramped, and conventional electrical humidity sensors cannot be used. Conventional electrical humidity sensors and hydrogen sensors should be calibrated regularly, which will change the condition of storage. Otherwise, the relative humidity of storage room is usually much lower than the limitation of normal hydrometer. It can be overcome easily by using LPFGs.

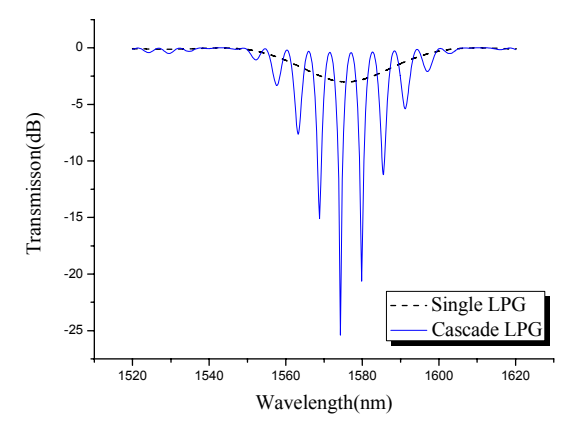

Fig. 3 Output spectrum of cascaded LFPGs.

When cascaded LFPGs are coated with hydrogel, the output spectrum of the sensor can be changed with different relative humidity as shown in Fig. 4 where wideband light source of super light emitting diode (SLED) is used. The humidity can be monitored by measuring either the phase term of interference or the fringe visibility associated with it. Both can be obtained by using Fourier analysis. Figure 5 shows the magnitude spectrum of the Fourier transform of measured spectrum for humidity values of $60 \%$ RH and 98\% RH, and Fig. 6 shows the variation of fringe visibility as a function of RH. Good linearity of the visibility of fringes has been obtained in upper humidity range from $60 \% \mathrm{RH}$ to $100 \% \mathrm{RH}$. The sensitivity and accuracy are measured to be as good as $9.9 \times 10^{-3} / \% \mathrm{RH}$ and $0.39 \% \mathrm{RH}$, respectively [4-5].

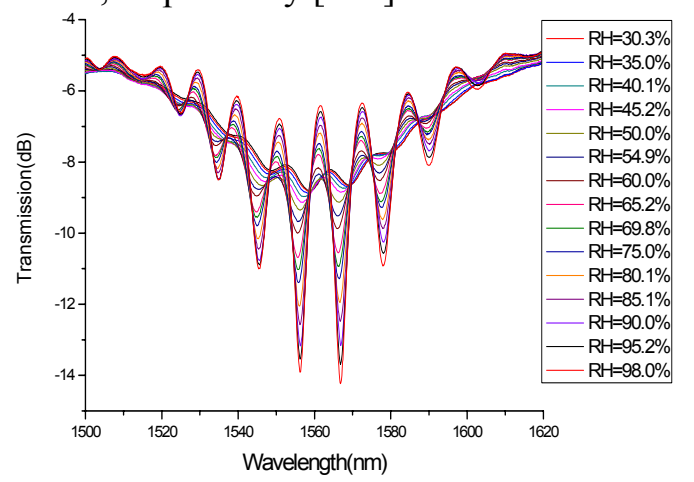

Fig. 4 Output spectrum of the sensor with different relative humidity. 


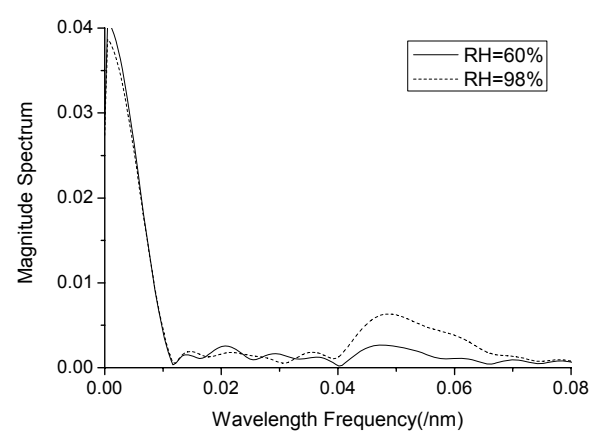

Fig. 5 Magnitude of Fourier transformation of measured spectrum with humidity values of $60 \% \mathrm{RH}$ and $98 \% \mathrm{RH}$.

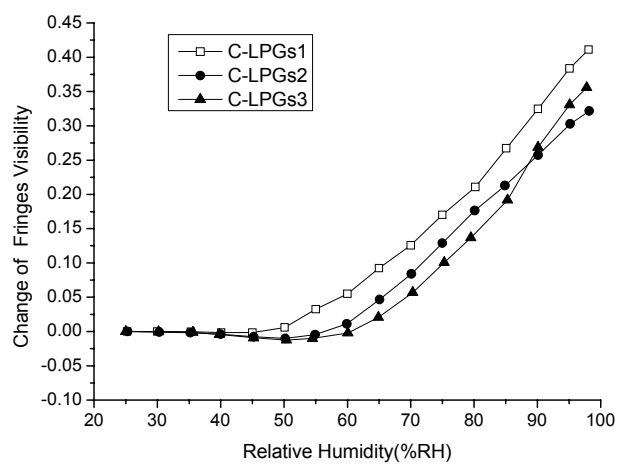

Fig. 6 Variation of fringe visibility as a function of RH.

By replacing the coating material with palladium silver film, hydrogen sensor can be fabricated. When Pd-Ag film absorbs hydrogen gas, the volume of film will expand and then change the period of cascaded LFPGs, which results in the variation of output spectrum. The concentration of hydrogen gas can then be calculated by using Fourier analysis of the output spectra. The hydrogen sensor can also be made by the use of fiber Fabry-Perot(FP) interferometer coated with $\mathrm{Pd}-\mathrm{Ag}$ film [6-7]. Both kinds of sensors are sensitive to temperature which should be compensated in operation.

In this area, there are still lots of research subjects can be done such as how to enlarge the linear range of sensor, how to increase to the responsibility of sensor by reducing the thickness or changing the mixture ratio of hydrogel or $\mathrm{Pd}-\mathrm{Ag}$ film.

\subsection{Spectrum analyzer for down-well oil detection}

It is quite important to distinguish the composition of liquid in crude oil well for oil exploration. The optical spectrum analyzer is the ideal way for distinguishing the components. But the condition down the oil well is severe for optical sensors with high temperature up to $175{ }^{\circ} \mathrm{C}$ and high atmospheric pressure of 20000 psi. Caustic gas such as $\mathrm{H}_{2} \mathrm{~S}$ and crude oil will also damage the fiber quickly.

Actually it is impossible to install a bulky optical spectrometer into the tube of the measurement system which is usually made from titanium alloy to protect optoelectronic components inside as shown in Fig. 7. An effective way is to use a discrete optical spectrometer by selecting several optimized wavelengths of the sample as shown in Fig. 8. Light from a wideband light source passes through the absorption cell and then is separated into several bunches of the fiber and received by photodiode in different wavelengths. The wavelength range of light is from $400 \mathrm{~nm}$ to $2000 \mathrm{~nm}$ and separated into 10 windows. Typical absorbtion windows of oil at $1720 \mathrm{~nm}$ and water at $1900 \mathrm{~nm}$ are used for calculating the concentration of oil and water and others are used to distinguish the quality of the sample obtained in deep well.

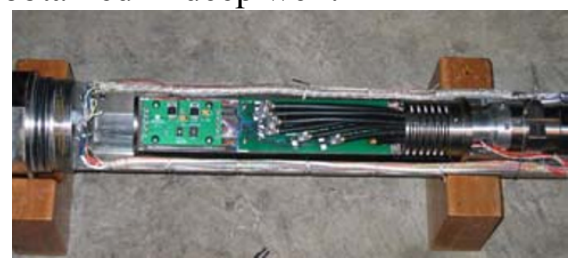

Fig. 7 Optical spectrometer in a titanium tube.

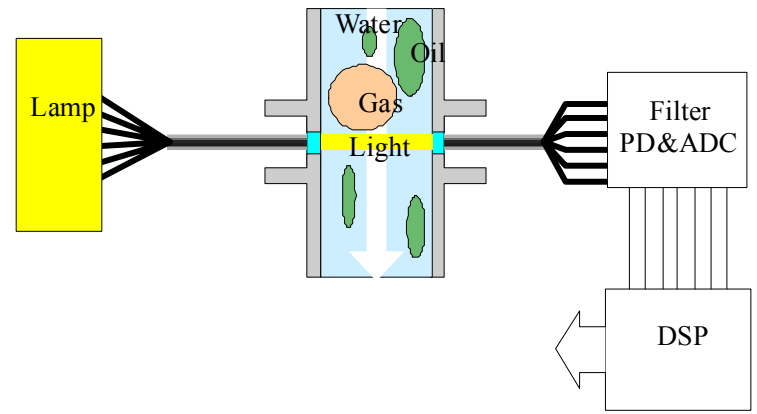

Fig. 8 Principle of the down well spectrometer.

Data then can be obtained by the acquisition system and transmitted to the recording system on the site. By using an algorithm of multiple 
regression analysis, the concentration of oil and water can be calculated. Special software has been developed as shown in Fig. 9 from CNOOC Company. Accuracy is measured to be as good as $1 \%$ which is enough for field exploration [8].

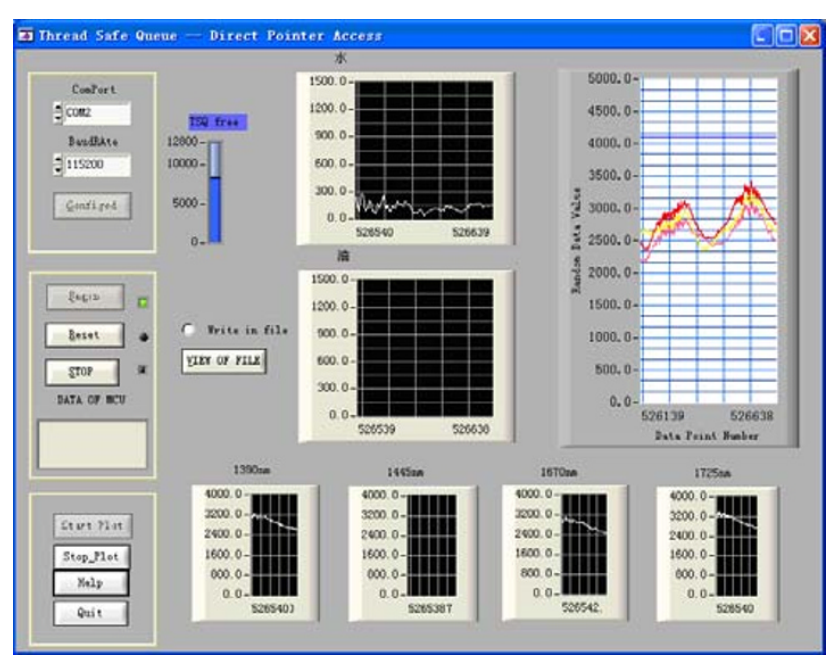

Fig. 9 Interface of the software for down well spectrometer.

\subsection{LIBS for heavy metal pollution detection}

With the development of modern industry and increasing life demands, lots of lands have suffered from pollution of heavy metal and pesticide contamination. There is still no effective way to monitor pollution directly in situ. The LIBS system may be one of ideal methods for quick detection of soil contamination.

In our project, a $Q$-switched YAG laser is used with energy of $20 \mathrm{~mJ}$ to $100 \mathrm{~mJ}$ and short pulse of $10 \mathrm{~ns}$ as shown in Figs. 10 and 11. The focused laser beam shots onto the sample. High peak power density $\left(>100 \mathrm{GW} / \mathrm{cm}^{2}\right)$ makes ultra high temperature $\left(10^{4} \mathrm{~K}\right)$ on the spot and drives it to the plasma state. Spectrum is then received by spectroscopy. By analyzing the characteristic atomic emission lines of the elements as shown in Fig. 12, the elements are identified and the concentration can be calculated. From the experiments, it is found that the background noise decreases with distance increasing from sparkle center, but signal spectrum intensity increases first and then decreases. By choosing the best detection position, high signal/background rate is obtained. To deal with complex spectrum and low signal/background rate problem, some algorithms have been carried out. By the use of Fourier's analysis, different parts of spectrum-white noise, thermal radiation, and emission spectral lines are studied. The emission spectral lines are separated from most of the noise, only missed a little white noise with band-pass filter. By calculating the similarity between detected line and its corresponding unit characteristic line, convolution intensity is introduced to measure the detected line intensity.

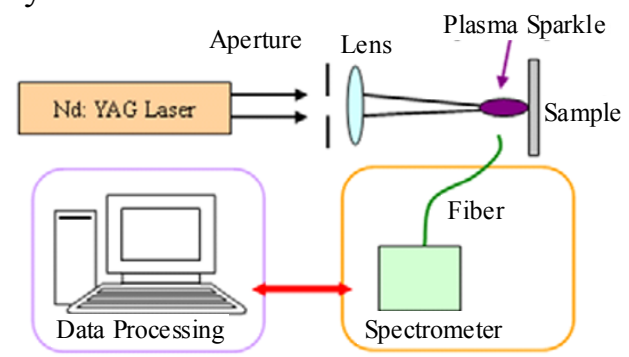

Fig. 10 Setup of LIBS system.

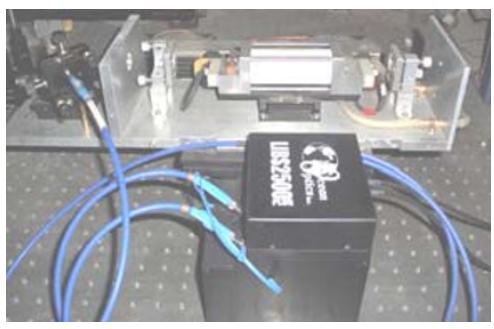

Fig. 11 YAG laser of LIBS.

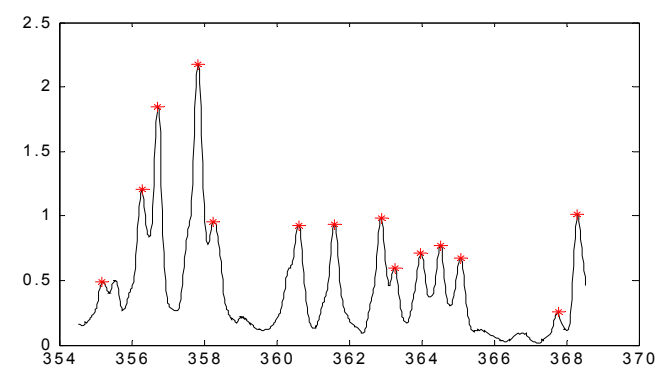

Fig. 12 LIBS spectrum.

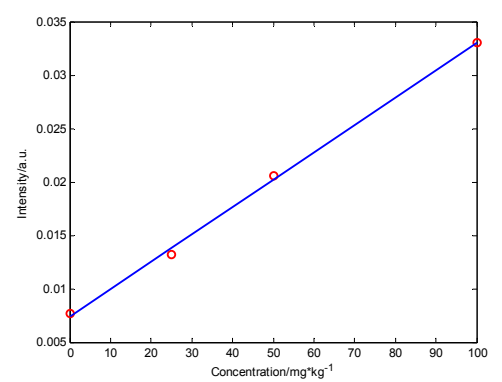

Fig. 13 Measured calibration curve of $\mathrm{Cd}$ in soil (Detection limit is $5 \mathrm{mg} / \mathrm{kg}$ ). 
The system has been used to detect $\mathrm{Cd}$ and $\mathrm{Cu}$ in soil. The detection limit of $5 \mathrm{mg} / \mathrm{kg}$ has been obtained for Cd detection as shown in Fig. 13, while the detection limitation of $\mathrm{Cu}$ is $42 \mathrm{mg} / \mathrm{kg}$ [9-11].

\section{Optical seismic geophone and its application}

\subsection{Introduction of seismic geophones}

The principle of most optical seismic geophones is based on fiber interferometer for its high sensitivity, large dynamic range, and wideband frequency response range. Several systems have been applied commercially, including the down well vertical seismic profile (VSP) seismic measurements and permanent fiber optic ocean bottom cable (OBC) systems which are now growing stronger due to the passive nature of the fiber system, with no subsea electronics, and hence in high reliability. Key technologies such as multi-wavelength laser sources with narrow line width of several kilohertz, optical couplers with high precise splitting ratio, and low bend loss fiber have been commercial and much cheaper, hence potential large scale multiplexing capability of OBC system as well as long range (10 s of km) can be realized [12]. But in early period of oil field exploration, the streamer cable with large scale is the only way for the detection in ocean. For such a large scale system, streamer cable with length of several kilometers is assembled one by one with lots of sections in $100 \mathrm{~m}$ long. The sensors and the circuits have to be installed in each section, where small size and high reliability are required. Figure 14 shows the optical fiber seismic hydrophones made in our lab for streamers and Fig. 15 shows the general system of optical seismic streamer cables.

Although requirements of streamers are quite different from $\mathrm{OBC}$ system, signal processing technologies of sensor are similar. We have developed a phase generate carrier (PGC) method by using Arctan algorithm with a look-up table to overcome the step response caused by gun source.
It has been realized by the use of field-programmable gate array (FPGA) demodulation system as shown in Fig. 16. The dynamic range of $130 \mathrm{~dB} / 100 \mathrm{~Hz}$ and the noise floor of $50 \mathrm{~dB} / \mu \mathrm{Pa}$ have been obtained [13-15].

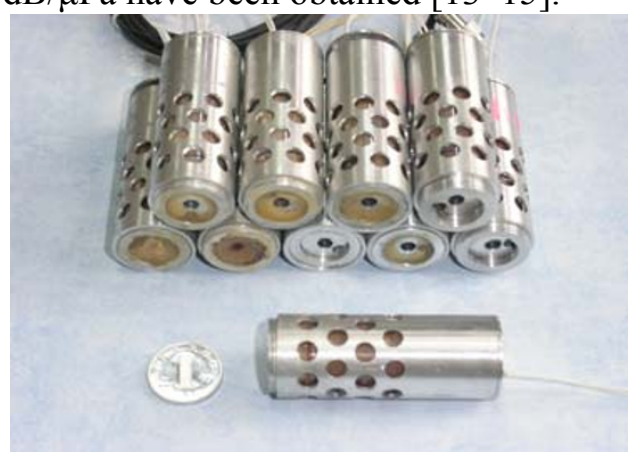

Fig. 14 Fiber seismic hydrophones.

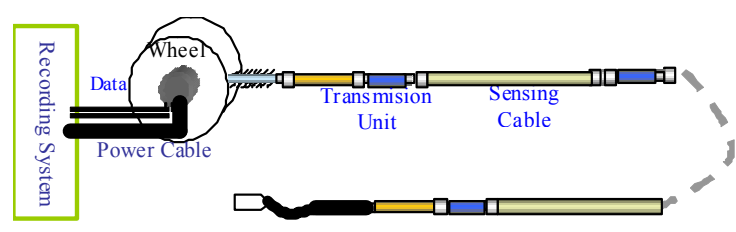

Fig. 15 System of the optical seismic streamer cable.

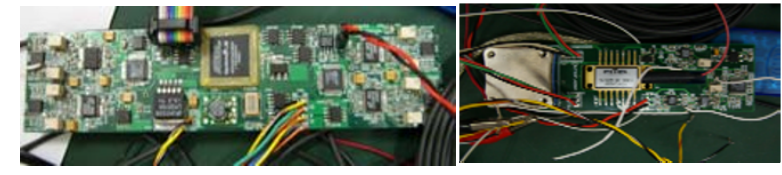

Fig. 16 Circuits of the PGC moduler and the laser driver.

\subsection{Field test of ocean oil seismic profile}

A practical system have been designed and tested. Figure 17 shows the seismic streamer cable rolled around the roller on the deck. Field experiments were done in Southern China Sea. Figure 18 shows the profile of the test field by processing the data obtained in experiments.

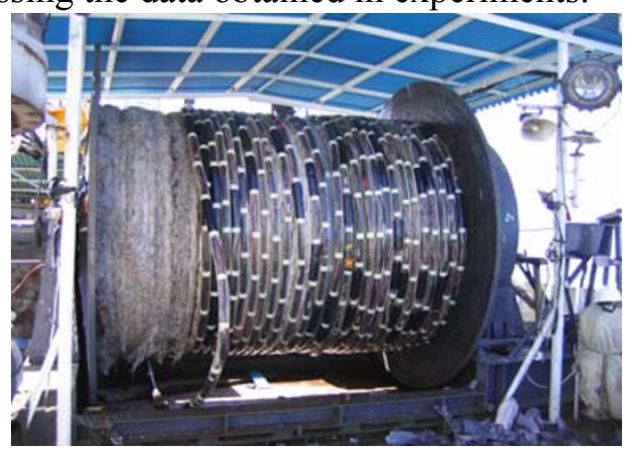

Fig. 17 Seismic streamer cable rolled on the wheel. 


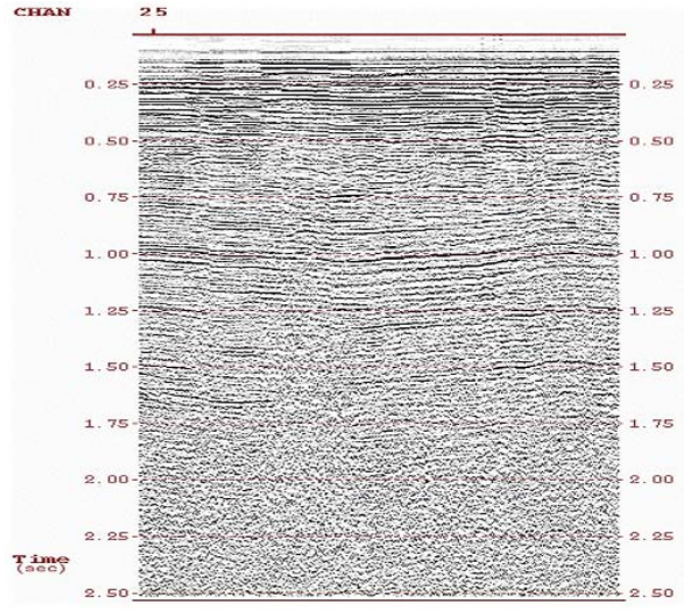

Fig. 18 Profile of the test field in Southern China Sea.

\section{Conclusions}

The interest of fiber sensors for oil and gas industry and other harsh environment is growing faster. It is believable that the market of fiber sensor will become larger with the development of relative technologies. We have designed several special sensors for harsh environment and investigated practical engineering applications. Further supports from government and industry are still necessary to push the development of optical fiber sensor in the future.

\section{Acknowledgment}

The work was supported by NSFC of China through grant 60629401 and 10776016, and also supported by the national 863 project of ocean area with grant number of 2006AA0AA102-03 and 863 project with grant number of 2006AA10Z209.

\section{References}

[1] Rao Yunjiang, Wang Yiping, Ran Zengling, et al. , "Novel fiber-optic sensors based on long-period fiber gratings written by high-frequency $\mathrm{CO}_{2}$ laser pulses," J. Lightwave Technol., vol. 52, no. 6, pp. 1432-1437, 2003.

[2] Liu Y, Wang L. W., Zhang M., et al., "Long-period grating relative humidity sensor with hydrogel coating," IEEE Photonics Technology Letters, vol. 19, no. 9-12, pp. 880-882, 2007.

[3] Yu X. J., Zhang M., Childs P., et al., "Research on testing the characteristics of hydrogel film by using long period fiber grating," Applied Optics, vol. 48, no. 11, pp. 2171-2177, 2009.
[4] Yu X. J., Childs P., Zhang M., et al., "Relative Humidity sensor based on cascaded long period gratings with hydrogel coatings and Fourier demodulation," IEEE Photonics Technology Letters, vol. 21, no. 24, pp. 1828-1830, 2009.

[5] Yu X. J., Childs P., Zhang M., et al., "Characteristics analysis of cascaded long-period gratings with nm-thick film coating," in Proceedings of SPIE, vol. 7508, pp. 75081N-75081N, 2009.

[6] Yang Z., Zhang M., Lei M., Yu X. J., et al., “An extrinsic Fabry-Perot interferometric optical fiber sensor system for hydrogen detection," in Proc. of SPIE, vol. 7503, pp.750323, 2009.

[7] Zhen Yang, Min Zhang, Yanbiao Liao, Shurong Lai, Qian Tian, Qisheng Li, Yi Zhang, and Zhi Zhuang, "A modified cross-correlation method for white-light optical fiber extrinsic Fabry-Perot interferometric hydrogen sensors," in Proc. of SPIE, vol. 7508, pp. 75081O, 2009

[8] Dehuan Meng, Xuan Haifeng, et al., "Performance analysis of an in-line optical fiber analysis system for well crude oil," in Proc. of SPIE, vol. 6595, pp. 65953S, 2007.

[9] Zheng Ze-ke, Ma Xiao-hong, Zhao Hua-feng, Yu Le, Zhang Min, and Liao Yan-biao, "Research on Laser Induced Breakdown Spectroscopy for Detection of Trace Cu in Polluted Soil," Spectroscopy and Spectral Analysis, vol. 29, no. 12, pp. 3383-3387, 2009.

[10] Qi Xu, XiaoHong Ma, and Huafeng Zhao, "Detection of sulfur dioxide in air by laser induced breakdown spectroscopy," presented at Proc. SPIE, Photonics Asia 2010, Beijing, Oct. 18-20, 2010.

[11] Yu Le, Zhao Hua-feng, Ma Xiao-hong, Liu Yang, Zhang Min, and Liao Yan-biao, "Research of LIBS method for detection of heavy metals in polluted soil," Laser Journal, vol. 29, no. 5, pp. 64-65, 2008( in Chinese).

[12] Hilde Nakstad and Jon Thomas Kringlebotn, "Optoplan AS, Realisation of a full-scale fibre optic ocean bottom seismic system," in 19th International Conference on Optical Fibre Sensors, Proc. of SPIE, vol, 7004, pp. 700436, 2008.

[13] Fukun Liao, Min Zhang, Liwei Wang, and Yanbiao Liao, "The Noise Analysis and Digital Realization of Arctangent Approach of PGC Demodulation for Optic Interferometric Sensors," in Proc. SPIE, vol. 6595, pp. 65954A, 2007.

[14] Pang M., Zhou H. P., Zhang M., Lin F., Zeng N., and Liao Y. B., "Analysis and amelioration about the cross-sensitivity of a fiber-optic accelerometer based on compliant cylinder," Journal of Lightwave Technology, vol. 26, no. 1-4, pp. 365-372, 2008.

[15] Shi Q. P., Tian Q., Wang L. W., et al., "Performance improvement of phase-generated carrier method by eliminating laser-intensity modulation for optical seismometer," Optical Engineering, vol. 49, no. 2, pp. 024402, 2010. 